\title{
Clinical Management of Adult Patients with a History of Nonsteroidal Anti-Inflammatory Drug-Induced Urticaria/ Angioedema: Update
}

\author{
Riccardo Asero, $M D$
}

In the large majority of previous studies, patients with a history of acute urticaria induced by nonsteroidal anti-inflammatory drugs (NSAIDs) seeking safe alternative drugs have undergone tolerance tests uniquely with compounds exerting little or no inhibitory effect on the cyclooxygenase 1 enzyme. In light of recently published studies, however, this approach seems inadequate and should be changed. The present article critically reviews the clinical management of patients presenting with a history of urticaria induced by a single NSAID or multiple NSAIDs and suggests a simple, updated diagnostic algorithm that may assist clinicians in correctly classifying their patients.

Key words: aspirin, drug allergy, nonsteroidal anti-inflammatory drug, urticaria

W onsteroidal anti-inflammatory drugs (NSAIDs) are 1 the most frequently prescribed drug class in the world. Their widespread use, further increased by the fact that, in many countries, some very popular compounds, such as acetylsalicylic acid (ASA), propionic acid derivatives, or paracetamol (acetaminophen), are present in over-the-counter drugs, is certainly the main cause for the increasing number of adverse reactions induced by these drugs that has been recorded worldwide. Although NSAIDs are generally well tolerated, they may induce a large spectrum of adverse reactions, some of which are potentially fatal. The most common adverse reactions linked to their inhibitory effects on the cyclooxygenase 1 (COX-1) enzyme are gastritis and peptic ulcers. Other adverse reactions include hepatitis and liver toxicity, anemia, interstitial nephritis, erythema multiforme, toxic epidermal necrolysis (Lyell's syndrome), Stevens-Johnson syndrome, and (cutaneous and/or respiratory) immediate allergic and pseudoallergic reactions. The term pseudoallergic defines reactions characterized by clinical symptoms that suggest an immune pathogenesis but for which there

Riccardo Asero: Ambulatorio di Allergologia, Clinica San Carlo, Paderno Dugnano (MI), Italy.

Correspondence to: Dr. Riccardo Asero, Ambulatorio di Allergologia, Clinica San Carlo, Via Ospedale 21, 20037 Paderno Dugnano (MI), Italy; e-mail: r.asero@libero.it.

DOI 10.2310/7480.2006.00018 is no evidence of an immune-mediated mechanism. ${ }^{1}$ Most pseudoallergic reactions to NSAIDs are presently considered to be associated with their inhibitory effects on the COX-1 enzyme. Urticaria/angioedema is the most common adverse reaction induced by NSAIDs seen by allergologists and probably represents the most frequent drug-induced skin disorder; it has been estimated that it occurs in 0.1 to $0.3 \%$ of subjects exposed to NSAIDs. ${ }^{2,3}$

One has to keep in mind that most patients presenting with an unequivocal history of urticaria (with or without angioedema) following the ingestion of NSAIDs are, reasonably, already convinced that they cannot take the offending drug any more. Invariably, their question is "What can I take in case of headache, pain, or fever?" The present article focuses on the clinical management of patients with NSAID-induced urticaria/angioedema in view of recently published literature. The present review was written on the basis of a literature search carried out using PubMed/MEDLINE. Articles dealing with NSAIDinduced urticaria published during the last 25 years were considered.

\section{Multiple- versus Single-NSAID Intolerance \\ Multiple-NSAID Intolerance}

It is well known that up to $30 \%$ of patients with chronic urticaria experience flares of hives following the ingestion of aspirin or chemically unrelated NSAIDs ${ }^{4-6}$; in general, 
offending drugs exert an inhibitory effect on the COX-1 enzyme. Unlike immunoglobulin (Ig)E-mediated hypersensitivity, this kind of intolerance frequently occurs on the first administration of a certain drug and parallels the clinical activity of the underlying chronic urticaria; drugs that induced severe skin reactions during a phase of moderate activity of the disease may be tolerated during a subsequent phase of remission.

Differently from chronic urticaria patients, the possible existence of otherwise normal subjects with multipleNSAID intolerance (defined as several distinct episodes of acute urticaria following the ingestion of chemically unrelated NSAIDs in the absence of any episode of spontaneous urticaria) has been a matter of debate for a long time. The 1998 edition of the most authoritative textbook of allergology still stated that "after earlier exposure to a specific ASA or NSAID, otherwise normalappearing individuals may develop urticaria, angioedema, or anaphylaxis on re-exposure to the same drug. In this type of reaction, cross-reactivity between ASA and NSAIDs does not occur." ${ }^{, 7}$ However, during the last two decades, a number of clinical studies assessing the tolerance to alternative NSAIDs in normal subjects with a history of single-NSAID intolerance found that some of them reacted to compounds that were chemically distinct from the offending ones and that were, hence, expected to be tolerated. ${ }^{8-15}$ Further, in one study specifically aiming to clarify this point, $36 \%$ of 261 subjects without chronic urticaria were finally found to have multiple-NSAID intolerance on the basis of the clinical history and oral tolerance test results. ${ }^{16}$ Interestingly, and similarly to patients with aspirin-exacerbated respiratory disease (AERD), in patients with acute urticaria induced by distinct NSAIDs (both with and without chronic urticaria), cross-reactions occurred mainly among COX-1inhibiting drugs, ${ }^{13,17}$ whereas drugs exerting little effect on the COX-1 enzyme (eg, nimesulide, paracetamol, COX-2 inhibitors) ${ }^{10,11,18-24}$ and NSAIDs characterized by different mechanisms of actions (floctafenine, paracetamol) or opiate agonists with analgesic activity (eg, tramadol) $)^{9,11,15,25}$ were generally well tolerated. These observations clearly suggested that COX-1 inhibition plays a pathogenic role in immediate pseudoallergic skin reactions induced by NSAIDs. COX blockade "deviates" arachidonic acid metabolism toward the 5-lipoxygenase pathway, and this eventually results in the production of cystinyl leukotrienes (Cys-LTs $\left.=\mathrm{LTC}_{4}, \mathrm{LTD}_{4}, \mathrm{LTE}_{4}\right)$. Cys-LTs are potent mediators of inflammatory processes, and there is some evidence that they may act as mediators in urticaria. Their intradermal injection elicits a wheal and flare reaction either in chronic urticaria patients or in normal subjects, ${ }^{26}$ and on a molar basis, Cys-LTs are 100 times more potent than histamine in inducing wheal and flare reactions. Recent studies showed that both chronic urticaria patients with NSAID intolerance and patients with AERD are characterized by elevated baseline urinary $\mathrm{LTE}_{4}$ levels and found that such levels are markedly increased by aspirin administration. ${ }^{27,28}$ The central role played by Cys-LTs as mediators of aspirin-induced urticaria (and probably of multiple-NSAID reactivity without chronic urticaria) is indirectly confirmed by studies showing a protective role by leukotriene receptor antagonists. ${ }^{29,30}$ Interestingly, several studies found an association between multiple-NSAID intolerance in otherwise normal subjects and atopic status. ${ }^{10,12,13,15}$

\section{Single-NSAID Intolerance}

Intolerance to single NSAIDs has been reported by several studies. Offending drugs include pyrazolones, ${ }^{24,31,32}$ paracetamol, ${ }^{33-38}$ aspirin, ${ }^{39}$ ketorolac, ${ }^{40}$ nimesulide, ${ }^{41}$ and celecoxib. ${ }^{42,43}$ It has been inferred that in a proportion of these cases, the pathogenesis is really IgE mediated, as sometimes suggested by positive skin tests with the offending compounds. Moreover, a genetic proneness to NSAID-induced anaphylactic reactions seems to exist. ${ }^{44}$ In patients with single-NSAID intolerance, cross-reactions may occur within the same chemical family but not between chemically distinct drugs, and this type of reaction never occurs on first exposition. However, the possibility that reactions to single NSAIDs are COX-1 mediated also cannot be ruled out. These patients might for some reason show a different threshold or different gene polymorphisms and develop multiple-NSAID intolerance at a later date. In effect, in a previous study, approximately $35 \%$ of otherwise normal patients with a history of urticaria induced by a single NSAID developed chronic urticaria 1 to 10 years after the adverse drug reaction, ${ }^{45}$ suggesting that chronic urticaria might remain in a state of latency for years, with NSAID intolerance as the only sign of its presence.

\section{New Classification of Immediate Allergic and Pseudoallergic NSAID-Induced Reactions}

Based on the studies reported above, in 2001, Stevenson and colleagues proposed a novel classification of allergic and pseudoallergic reactions induced by NSAIDs that includes six distinct categories of patients (Table 1). ${ }^{46}$ Interestingly, skin reactions (urticaria/angioedema) are 
Table 1. Classification of Allergic and Pseudoallergic Reactions Induced by Nonsteroidal Anti-Inflammatory Drugs

\begin{tabular}{|c|c|c|c|}
\hline & Type of Allergic/Pseudoallergic Reactions & Underlying Disorder & $\begin{array}{c}\text { Cross-Reaction/Reaction on } \\
\text { First Exposure }\end{array}$ \\
\hline 1 & Asthma and rhinitis exacerbated by NSAID & Asthma/sinusitis/polyposis & Yes \\
\hline 2 & Urticaria/angioedema exacerbated by NSAID & Chronic urticaria & Yes \\
\hline 3 & Urticaria/angioedema from single NSAID & None & No \\
\hline 4 & Acute urticaria/angioedema from multiple NSAIDs & None & Yes \\
\hline 5 & Anaphylaxis from single NSAID & None & No \\
\hline 6 & $\begin{array}{l}\text { Blended respiratory/cutaneous reaction from one } \\
\text { or more NSAIDs }\end{array}$ & Asthma/rhinitis/polyposis or none & Yes or No \\
\hline
\end{tabular}

NSAID $=$ nonsteroidal anti-inflammatory drug.

present in five of six categories. This classification was subsequently adopted in the last edition of the treatise Allergy: Principles and Practice. ${ }^{47}$ Notably, the difference between type 2 and type 4 multiple-NSAID intolerance is based uniquely on the presence or absence of chronic urticaria as an underlying disorder. Recent studies seem to abolish even this distinction as

1. type 4 subjects show an extremely high prevalence of positive reactions on an autologous serum skin test, ${ }^{48} \mathrm{a}$ typical feature of patients with autoreactive chronic urticaria. A positive autologous serum skin test has been associated with circulating IgG autoantibodies specific for IgE or for the high-affinity IgE receptor FceRI, present on basophils and mast cells ${ }^{49}$

2. approximately $35 \%$ of otherwise normal patients with a history of single- or multiple-NSAID intolerance (urticaria) develop chronic urticaria 1 to 10 years after the adverse drug reaction ${ }^{45}$

\section{Diagnostic Workup}

In view of the possible distinct pathogenesis underlying multiple- or single-NSAID reactivity, the most important clinical point to establish is whether the patient presenting with a history of NSAID-induced urticaria/angioedema is a monoreactor or a multireactor. To this end, both a thorough interview and oral challenge tests with properly chosen alternative substances are essential. A classification of the most important NSAIDs according to their inhibitory effect on COX isoenzymes is shown in Table 2 .

A confirmative provocation test with the reported offending drug is not warranted for the following reasons:

1. In monosensitized patients with IgE-mediated hypersensitivity, the challenge test might cause severe, even life-threatening adverse reactions.
2. In the clinical practice, the offending drug can, in most instances, be substituted with a number of equally effective but chemically distinct compounds.

In patients with a history of urticaria/angioedema caused by a single COX-1 inhibitor (eg, diclofenac, piroxicam, naproxen, aspirin), tolerance tests should start with a chemically distinct COX-1 inhibitor. There are several reasons why these patients should be challenged first with another nonselective COX inhibitor rather than with a selective COX-2 inhibitor. First, this is the only way to establish whether the patient is really monosensitized (ie, if the patient may take any NSAID other than the offending one) or if the reported reaction represents the first sign of a multiple-NSAID intolerance. Second, the long-term use of COX inhibitors has been associated with

Table 2. Classification of the Most Commonly Employed NSAIDs According to Their Inhibitory Effect on COX Isoenzymes

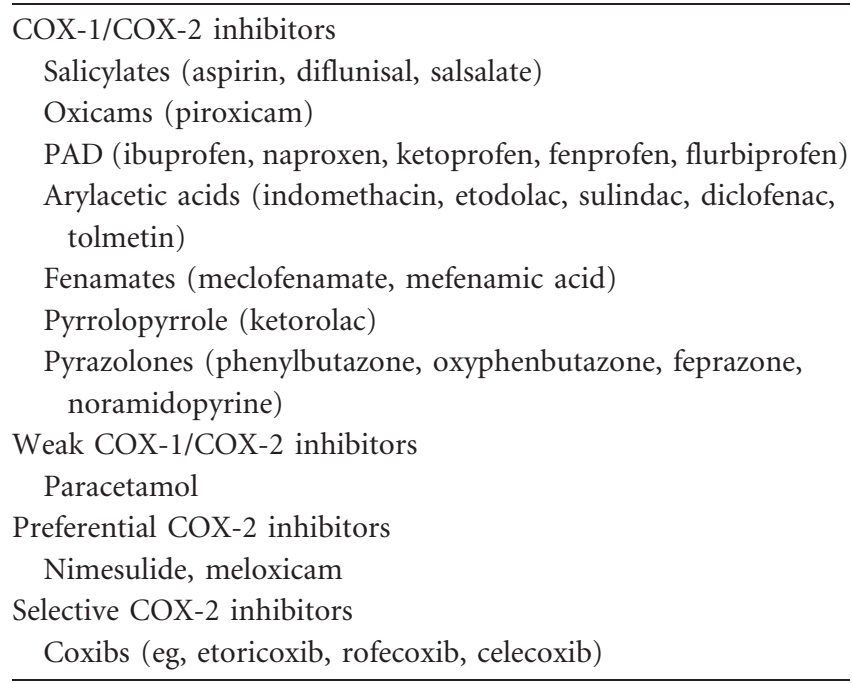

$\mathrm{COX}=$ cyclooxygenase; NSAID $=$ nonsteroidal anti-inflammatory drug $\mathrm{PAD}=$ propionic acid derivatives. 
an increase in cardiovascular events, ${ }^{50}$ and this has brought about the withdrawal of most of them from the market; presently, the only surviving drug of this class is etoricoxib, which is, however, under examination by governmental drug agencies. Similarly, floctafenine was withdrawn from the market some years ago. As a consequence, the spectrum of NSAIDs exerting little or no inhibitory activity on COX-1 is presently very limited, including only nimesulide, paracetamol, and meloxicam. Third, the anti-inflammatory and/or analgesic activity of these remaining substances (nimesulide, paracetamol, meloxicam) is, in most cases, inferior to nonselective COX inhibitors and not sufficient to control adequately chronic inflammatory disorders, such as arthritis.

If the alternative COX-1 enzyme-inhibiting drug is tolerated, the patient is diagnosed as having single-drug intolerance, and no further tests are needed. In contrast, intolerance to the challenged drug suggests multiple-NSAID intolerance, and further challenges with drugs exerting little or no inhibitory activity on the COX-1 enzyme (eg, nimesulide, coxibs, paracetamol, tramadol) should be performed to detect at least some tolerated drugs. Two very recent studies clearly address these aspects. In the first one, only 28 of 117 (24\%) otherwise normal subjects with a history of acute urticaria induced by a single NSAID other than aspirin did not tolerate aspirin on single-blind, placebo-controlled oral challenges, with no differences between patients reactive to different NSAIDs; 5 of these $28(18 \%)$ subjects also did not tolerate NSAIDs exerting little or no inhibitory activity on the COX-1 enzyme on subsequent oral challenges. ${ }^{51}$ In the second study, of 40 otherwise normal subjects with a history of acute urticaria following the ingestion of aspirin, $24(60 \%)$ did not tolerate ketoprofen on single-blind, placebo-controlled oral challenges. ${ }^{52}$ On subsequent challenges, 3 of 8 (37\%) ketoprofen reactors did not tolerate nimesulide. Three ketoprofen reactors reported the onset of spontaneous recurrent urticaria 1 to 3 years after the challenge tests. The choice of aspirin and ketoprofen as challenged substances in these two studies is based on their ranking among the strongest COX-1 inhibitors. ${ }^{53}$ These two studies show that oral challenges with alternative COX-1 inhibitors are essential to establish whether subjects with a history of urticaria induced by a single NSAID are really single-NSAID reactors; in these subjects, weak COX-1 inhibitors should be challenged subsequently only in the case of multiple-NSAID intolerance. One further interesting observation coming from these studies is that patients with a history of aspirininduced urticaria seem more prone to develop multipleNSAID intolerance than patients with a history of urticaria induced by another NSAID ( $60 \%$ vs $24 \%$ ). This finding is in keeping with the observations of another study that both multiple- and single-NSAID reactors with a history of aspirin-induced urticaria seem at higher risk of chronic urticaria than patients with a history of single intolerance to NSAIDs other than aspirin. ${ }^{45}$

Patients already presenting with a history of multipleNSAID intolerance, with or without underlying chronic urticaria, should directly undergo oral tolerance tests with drugs exerting little or no COX-1 inhibition. ${ }^{53}$

In patients with chronic urticaria, a state of moderate activity of the underlying disease will probably avoid falsenegative results. In these patients, it is also essential that the challenged drug induces an unequivocal exacerbation of underlying urticaria to produce a positive result. In doubtful cases, patients with active urticaria should be challenged a second time to confirm that any reaction or exacerbation is truly due to the drug being tested.

Finally, in patients with a history of an allergic or anaphylactic reaction to ASA who need aspirin as a prophylactic treatment for coronary artery disease or for angioplasty or stent procedures, the safest procedure is probably to give alternative prophylactic substances, such as indobufen, ticlopidine, clopidogrel, or dipyridamole.

The suggested diagnostic workup is shown in Figure 1. Since evidence that patients with a history of NSAIDinduced anaphylaxis may cross-react to chemically unrelated NSAIDs is lacking, such patients should be managed exactly as those with a history of urticaria induced by a single NSAID. In this sense, the proposed algorithm simplifies that previously suggested by Sanchez-Borges and colleagues. ${ }^{54}$

\section{Oral Tolerance/Provocation Tests}

Practically, oral tolerance/provocation challenges are carried out, giving patients increasing doses of the drug under consideration until the therapeutic dose is reached. In general, based on previous studies from this allergy centre, ${ }^{14-16,41,51,52}$ two doses per substance (corresponding to one-quarter and three-quarters of a therapeutic dose) given at 1-hour intervals seem to be a safe, convenient, and sensitive way to detect multiple-NSAID intolerance. Patients should be kept under observation for at least 1.5 hours after the last provocative ${ }^{55}$ dose as most adverse reactions occur within this short time. In otherwise normal subjects (ie, patients without a history of chronic urticaria), oral tolerance tests can be carried out in an open fashion. In subjects with chronic urticaria, it might be necessary to carry out these tests in a single-blind, 


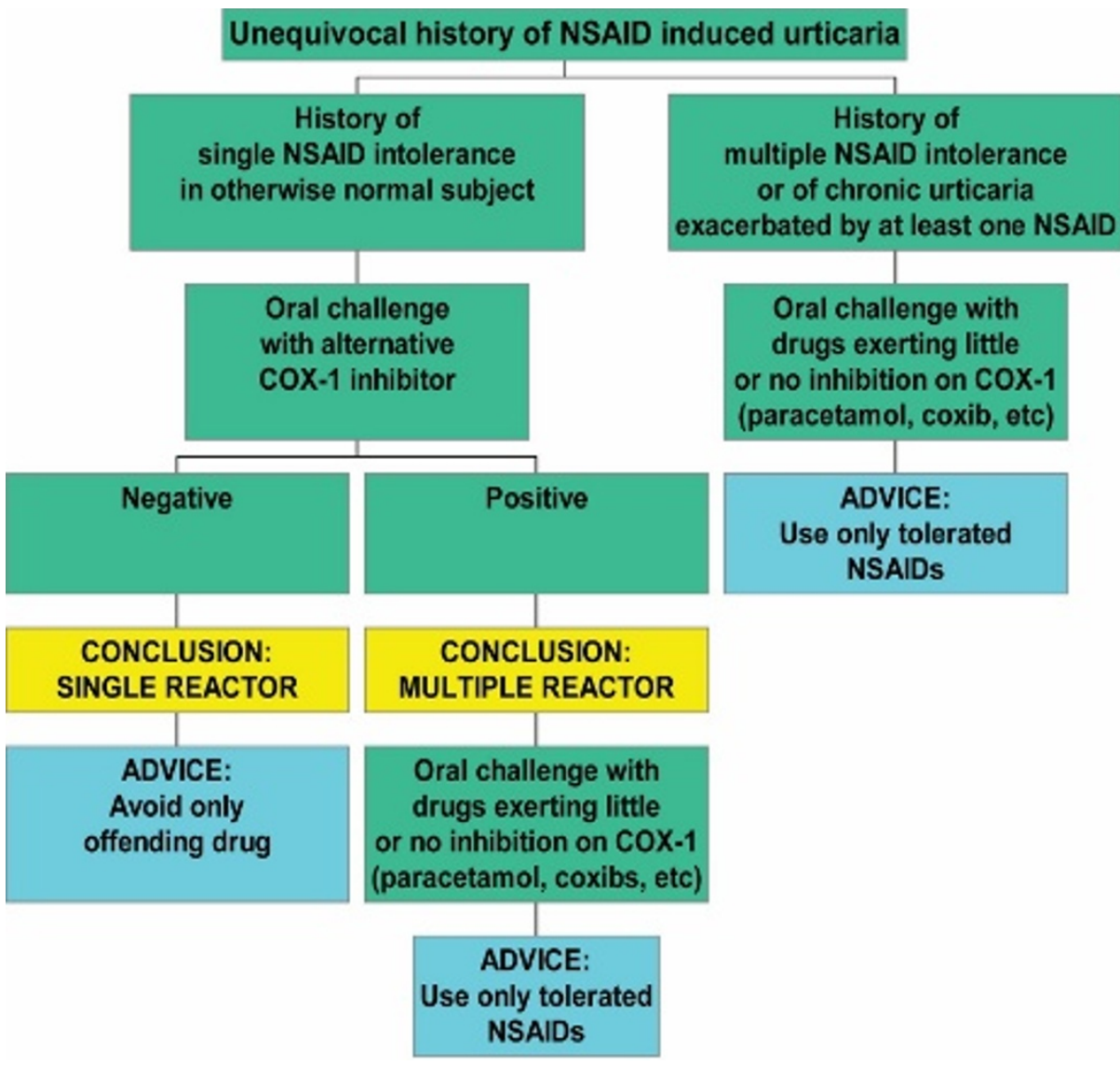

Figure 1. Diagnostic workup in patients intolerant to nonsteroidal anti-inflammatory drugs (NSAID). $\mathrm{COX}=$ cyclooxygenase. placebo-controlled manner. Only the appearance of unequivocal urticaria/angioedema should be considered a positive response.

\section{Conclusion}

In the absence of reliable in vivo and in vitro tests, oral challenge tests remain the only way to assess tolerance or intolerance to specific NSAIDs in subjects with a history of urticaria induced by these substances and, hence, to respond satisfactorily to patients' requests and needs. Progress in the knowledge of the pathogenesis of immediate allergic and pseudoallergic reactions induced by NSAIDs, along with the observations coming from recent studies of oral challenges with alternative anti-inflammatory drugs, has led to a simplification of our approach to patients with a history of NSAID-induced urticaria.

\section{References}

1. Adkinson NF. Drug allergy. In: Middleton E, Reed CE, Ellis EF, et al, editors. Allergy: principles and practice. 5th ed. St Louis: CV Mosby; 1998. p. 1212-24.
2. Settipane GA. Aspirin and allergic diseases. Am J Med 1983;74: 102-10.

3. Strom BL, Carson JL, Lee Morse M, et al. The effect of indication on hypersensitivity reactions associated with zomepirac sodium and other nonsteroidal anti-inflammatory drugs. Arthritis Rheum 1987;30:1142-8.

4. Settipane RA, Constantine HP, Settipane GA. Aspirin intolerance and recurrent urticaria in normal adults and children. Allergy 1980;35:149-54.

5. Mathison DA, Lumry WS, Stevenson DD, Curd JG. Aspirin in chronic urticaria and/or angioedema: studies of sensitivity and desensitisation [abstract]. J Allergy Clin Immunol 1982;69 Suppl: 135.

6. Stevenson DD, Simon RA. Sensitivity to aspirin and nonsteroidal anti-inflammatory drugs. In: Middleton E, Reed CE, Ellis EF, et al, editors. Allergy: principles and practice. 4th ed. St Louis: CV Mosby; 1993. p. 1747-65.

7. Stevenson DD, Simon RA. Sensitivity to aspirin and nonsteroidal anti-inflammatory drugs. In: Middleton E, Reed CE, Ellis EF, et al, editors. Allergy: principles and practice. 5th ed. St Louis: CV Mosby; 1998. p. 1225-34.

8. Szczeklik A. Adverse reactions to aspirin and nonsteroidal antiinflammatory drugs. Ann Allergy 1987;57:113-8.

9. Papa G, Romano A, Del Bono A, et al. Floctafenine: a valid alternative in patients with adverse reactions to nonsteroidal anti-inflammatory drugs. Ann Allergy Asthma Immunol 1997;78: 74-8. 
10. Pastorello E, Zara C, Riario-Sforza GG, et al. Atopy and intolerance of antimicrobial drugs increase the risk of reactions to acetaminophen and nimesulide in patients allergic to nonsteroidal antiinflammatory drugs. Allergy 1998;53:880-4.

11. Quaratino D, Romano A, Papa G, et al. Long-term tolerability of nimesulide and acetaminophen in nonsteroidal anti-inflammatory drug-intolerant patients. Ann Allergy Asthma Immunol 1997;79: 47-50.

12. Quiralte J, Blanco C, Castillo R, et al. Intolerance to nonsteroidal anti-inflammatory drugs: results of controlled drug challenges in 98 patients. J Allergy Clin Immunol 1996;98:67885 .

13. Sanchez-Borges M, Capriles-Hulett A. Atopy is a risk factor for nonsteroidal anti-inflammatory drug sensitivity. Ann Allergy Asthma Immunol 2000;84:101-6.

14. Asero R. Detection of aspirin reactivity in patients with pyrazolones-induced skin disorders. Allergy 1998;53:214-5.

15. Asero R. Risk factors for acetaminophen and nimesulide intolerance in patients with NSAID-induced skin disorders. Ann Allergy Asthma Immunol 1999;82:554-8.

16. Asero R. Multiple sensitivity to NSAID. Allergy 2000;55:893-4.

17. Carmona MJ, Blanca M, Garcia A, et al. Intolerance to piroxicam in patients with adverse reactions to nonsteridal antiinflammatory drugs. J Allergy Clin Immunol 1992;90:873-9.

18. Andri L, Senna G, Betteli C, et al. Tolerability of nimesulide in aspirin-sensitive patients. Ann Allergy 1994;72:29-32.

19. Ispano M, Fontana A, Scibilia J, Ortolani C. Oral challenge with alternative nonsteroidal anti-inflammatory drugs (NSAIDs) and paracetamol in patients intolerant to these agents. Drugs 1993;46 Suppl:253-6.

20. Sanchez-Borges M, Capriles-Hulett A, Caballero-Fonseca F, Perez CR. Tolerability to new COX-2 inhibitors in NSAIDsensitive patients with cutaneous reactions. Ann Allergy Asthma Immunol 2001;87:201-4.

21. Asero R. Tolerability of rofecoxib. Allergy 2001;56:916-7.

22. Nettis E, Di Paola R, Ferrannini A, Tursi A. Tolerability of rofecoxib in patients with cutaneous adverse reactions to nonsteroidal anti-inflammatory drugs. Ann Allergy Asthma Immunol 2002;88:331-4.

23. Pacor ML, Di Lorenzo G, Biasi D, et al. Safety of rofecoxib in subjects with a history of adverse cutaneous reactions to aspirin and/or nonsteroidal anti-inflammatory drugs. Clin Exp Allergy 2002;32:397-400.

24. Nettis E, Di Paola R, Ferrannini A, et al. Meloxicam in hypersensitivity to NSAIDs. Allergy 2001;56:803-4.

25. Asero R. Chronic urticaria with multiple NSAID intolerance: is tramadol always a safe alternative analgesic? J Invest Allergol Clin Immunol 2003;13:55-8.

26. Maxwell DL, Atkinson BA, Spur BW, et al. Skin responses to intradermal histamine and leukotrienes $\mathrm{C} 4, \mathrm{D} 4$, and $\mathrm{E} 4$ in patients with chronic idiopathic urticaria and in normal subjects. J Allergy Clin Immunol 1990;86:759-65.

27. Mastalerz L, Setkowicz M, Sanak M, Szczeklik A. Hypersensitivity to aspirin: common eicosanoid alterations in urticaria and asthma. J Allergy Clin Immunol 2004;113:771-5.

28. Mastalerz L, Setkowicz M, Szczeklik A. Mechanism of chronic urticaria exacerbation by aspirin. Curr Allergy Asthma Rep 2005;5: 277-83.
29. Asero R. Leukotriene receptor antagonists may prevent NSAIDinduced exacerbations in patients with chronic urticaria. Ann Allergy Asthma Immunol 2000;85:156-7.

30. Perez C, Sanchez-Borges M, Capriles E. Pretreatment with montelukast blocks NSAID-induced urticaria and angioedema. J Allergy Clin Immunol 2001;108:1060-1.

31. Czerniawska-Mysik G, Szczeklik A. Idiosyncrasy to pyrazolone drugs. Allergy 1981;36:381-4.

32. Quiralte J, Blanco C, Castillo R, et al. Anaphylactoid reactions due to nonsteroidal anti-inflammatory drugs: clinical and crossreactivity studies. Ann Allergy Asthma Immunol 1997;78:293-6.

33. Ownby DR. Acetaminophen-induced urticaria and tolerance of ibuprofen in an eight-year-old child. J Allergy Clin Immunol 1997; 99:151-2.

34. Vidal C, Perez-Carral C, Gonzalez-Quintela A. Paracetamol (acetaminophen) hypersensitivity. Ann Allergy Asthma Immunol 1997;79:320-1.

35. Mendizabal SL, Diez-Gomez ML. Paracetamol sensitivity without aspirin intolerance. Allergy 1998;53:457-8.

36. Kivity S, Pawlik I, Katz J. Acetaminophen hypersensitivity. Allergy 1999;54:187-8.

37. De Paramo BJ, Gancedo SQ, Cuevas $M$, et al. Paracetamol (acetaminophen) hypersensitivity. Ann Allergy Asthma Immunol 2000;85:508-11.

38. Grant JA, Weiler JM. A report of a rare immediate reaction after ingestion of acetaminophen. Ann Allergy Asthma Immunol 2001; $87: 227-9$

39. Blanca M, Perez E, Garcia JJ, et al. Angioedema and IgE antibodies to aspirin: a case report. Ann Allergy 1989;62:295-8.

40. Scala E, Giani M, Pirrotta L, et al. Selective severe anaphylactic reaction due to ketorolac tromethamine without nonsteroidal antiinflammatory drug intolerance. J Allergy Clin Immunol 2001;107: 557.

41. Asero R. Aspirin and paracetamol tolerance in patients with nimesulide-induced urticaria. Ann Allergy Asthma Immunol 1998; 82:237-8.

42. Levy MB, Fink JN. Anaphylaxis to celecoxib. Ann Allergy Asthma Immunol 2001;87:72-3.

43. Grob M, Pichler WJ, Wuthrich B. Anaphylaxis to celecoxib. Allergy 2002;57:264-5.

44. Quiralte J, Sanchez-Garcia F, Torres MJ, et al. Association of HLA-DR11 with the anaphylactoid reaction caused by nonsteroidal anti-inflammatory drugs. J Allergy Clin Immunol 1999;103: $685-9$.

45. Asero R. Intolerance to nonsteroidal anti-inflammatory drugs might precede by years the onset of chronic urticaria. J Allergy Clin Immunol 2003;111:1095-8.

46. Stevenson DD, Sanchez-Borges M, Szczeklik A. Classification of allergic and pseudoallergic reactions to drugs that inhibit cyclooxygenase enzymes [editorial]. Ann Allergy Asthma Immunol 2001;87:1-4.

47. Stevenson DD, Simon RA, Zuraw BL. Sensitivity to aspirin and nonsteroidal anti-inflammatory drugs. In: Adkinson NF, Yunginger JW, Busse WW, et al, editors. Allergy: principles and practice. 6th ed. St Louis: CV Mosby; 2003. p. 1695-710.

48. Asero R, Tedeschi A, Lorini M. Autoreactivity is highly prevalent in patients with multiple intolerances to NSAID. Ann Allergy Asthma Immunol 2002;88:468-72. 
49. Sabroe RA, Grattan CE, Francis DM, et al. The autologous serum skin test: a screening test for autoantibodies in chronic idiopathic urticaria. Br J Dermatol 1999;140:446-52.

50. Mukerjee D, Nissen SE, Topol EJ. Risk of cardiovascular events associated with selective COX-2 inhibitors. JAMA 2001;286:954-9.

51. Asero R. Oral aspirin challenges in patients with a history of intolerance to single non-steroidal anti-inflammatory drugs. Clin Exp Allergy 2005;35:713-6.

52. Asero R. Use of ketoprofen oral challenges to detect cross-reactors among patients with a history of aspirin-induced urticaria. Ann Allergy Asthma Immunol 2006;97:187-9.
53. Warner TD, Giuliano F, Vojnovic I, et al. Nonsteroid drug selectivities for cyclo-oxygenase 1 rather than cyclo-oxygenase 2 are associated with human gastrointestinal toxicity: a full in vitro analysis. Proc Natl Acad Sci U S A 1999;96:7563-8.

54. Sanchez-Borges M, Capriles-Hulett A, Caballero-Fonseca F. NSAID-induced urticaria and angioedema: a reappraisal of its clinical management. Am J Clin Dermatol 2002;3:599-607.

55. Cormican LJ, Farooque S, Altmann DR, Lee TH. Improvements in an oral aspirin challenge protocol for the diagnosis of aspirin hypersensitivity. Clin Exp Allergy 2005;35:71722 . 